\title{
Madelung Disease - Our Clinical Considerations and Management
}

\author{
Toader Corneliu ${ }^{1}$, Stroi Marian ${ }^{1}$, Sinelli Joanna $I^{1}$ and Boghi David $\mathbf{M}^{* 2}$ \\ ${ }^{1}$ National Institute for Neurology and Neuro-vascular diseases, Bucharest, Romania \\ 2"Saint Pantelimon" Clinical Hospital, Bucharest, Romania \\ *Corresponding author: Boghi David Matteo, "Saint Pantelimon" Clinical Hospital, Bucharest, Romania
}

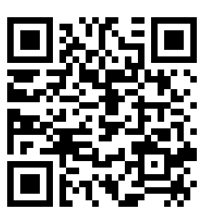

\section{ARTICLE INFO}

Received: 㓞 January 19, 2021

Published: 幽 January 26, 2021

Citation: Toader Corneliu, Stroi Marian, Sinelli Joanna I, Boghi David M. Madelung Disease - Our Clinical Considerations and Management. Biomed J Sci \& Tech Res 33(3)-2021. BJSTR. MS.ID.005397.

\section{ABSTRACT}

Although Madelung Disease (MD) is a quite rare clinical entity, it may have some serious concerns regarding the patient's quality of life. Such lesions are painless, however the exterior aspect may lead to social ostracism. The physio-pathological processes involved are not fully understood, however male sex and chronic alcohol consumption have been described as risk-factors. We hereby present our case of a middle-age man who came in our Hospital with the clinical complaint of a painless bulging masses on the posterior aspect of the neck, with extension into the occipital area. The mass grew in a period of almost 4 years and was considered trivial by the patient. The clinical examination and the imagistic studies confirmed the presence of symmetrical lipomatous deposits. We provided a surgical operation, consisting of a bilateral ablation of the two formations. The postoperative out-come was positive, with an optimal esthetical result achieved.

\section{Introduction}

Madelung disease, or Multiple Symmetric Lipomatosis (MSL) is a rare disturbance among the overgrowth syndromes and it is characterized by painless, symmetric and non-encapsulated fatty deposits in varies district of the body, including the cervical region, limbs, torso, neck, mammary and abdominal regions. The etiology of this disease is still not well elucidated and its risk factors are chronic alcoholic consumption and male sex. The chronical alcoholic consumption may play a role in adipocytes hyperplasia in genetically susceptible patients, however is not fully understood yet. Such kind of patients usually seek medical attention for esthetic reasons.

\section{Case-Presentation}

Here we report our case of a 37-year old male patient, without any relevant clinical history, which came in our hospital for this painless, bilateral and symmetric growing mass on the posterior aspect of the neck region, which grew in the last 4 years and speed up in the last year. At the beginning, the lesion was considered trivial by the patient. His main complaint was esthetical. At the physical examination, two well distinct lesions were described, as the left side was slightly bigger than the right side $(10 \mathrm{~cm} ; 8 \mathrm{~cm}$ as thier biggest diameter) and without any invasion of the skin layers. We also provided the patient with a CT scan of the head and neck regions (Figure 1), which confirmed the adipocytic origin of the tumor, as well as its lacks of infiltration of other compartments. There were no sign of airway obstruction or involvement of the superior aspect of mediastinum. The lesion was well delimitated and encapsulated.

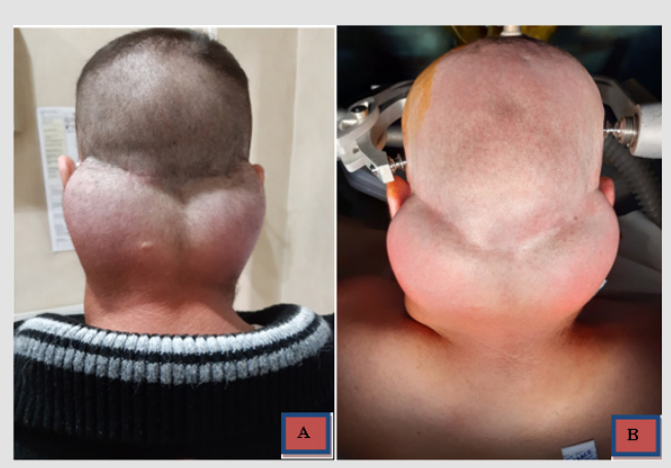

Figure 1:

A. Macroscopic aspect of the lesions.

B. The patient is set in the Mayfield support. 
As other complaints, he presented with type I mild obesity. He denied a daily alcoholic intake, but he confirmed its sporadic use. No other relevant disease or disturb was found through the clinical examination. The physical examination also revealed that the two masses were quite hard at touch, relatively firm in position and only in contact with the posterior aspect of the neck. No cervical vein engorgement was described (Figure 2) A one-step surgical treatment was scheduled a few days later after the patient came in. The surgical procedure consisted of a macroscopic ablation of the two formations through a bilateral vertical incisure and ablation was performed under general anesthesia and mechanic ventilation. The operation lasted circa 3 hours. After the operation the patient was satisfied and was discharged a few days later. No complications were encountered. The esthetical result was satisfactory.
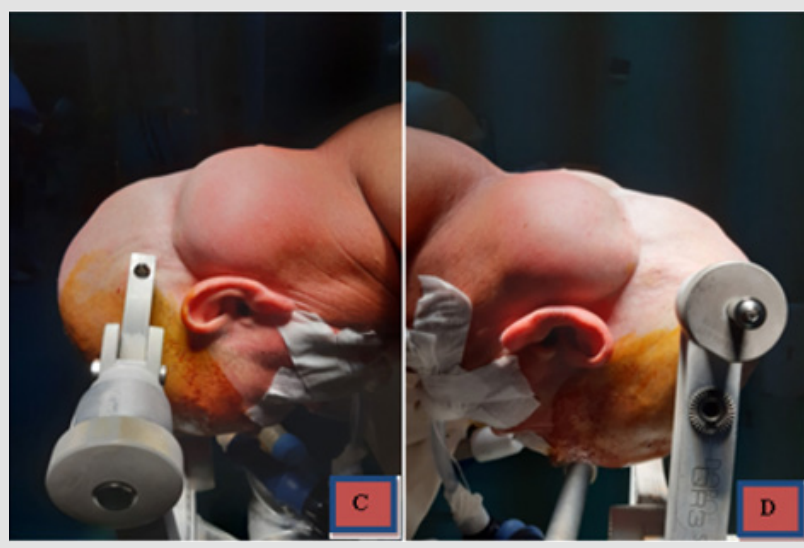

Figure 2:

C. Lefts side of the patient.

D. Right side of the patient.

\section{Materials and Methods}
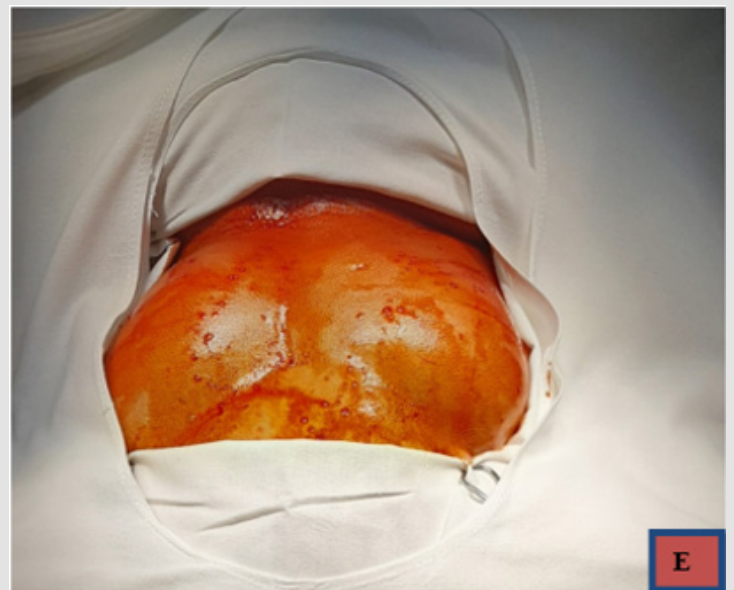

Figure 3: Surgical field.

A full clinical examination of the patient was performed. The patient denied any chronic alcoholic consumption. No sign of any other chronic condition has been found. He did not present diabetes mellitus or other metabolic disorder. Patient's BMI was calculated as 24.1 (slightly overweight). However, the patient could describe a similar case in his own familiar tree, as his grand-father had a similar condition. We performed a surgical macroscopical ablation of the tumors, using a Mayfield Head support (Figures 3) for the surgical table, and the patient was given general anesthesia, mechanical ventilation and the patient was in prone position (Figure 4), parallel with the ground and the neck was slighlty flexed (circa 15 degrees from ground). We made two incisions in the skin (Figure 5), perpendicular to the spinal column and circa $5 \mathrm{~cm}$ in lenght. We operated first the right formation and then the left one in a single step surgery. Dissection was performed with monopolar coagulation device and by free-hand by the Attending Surgeon.

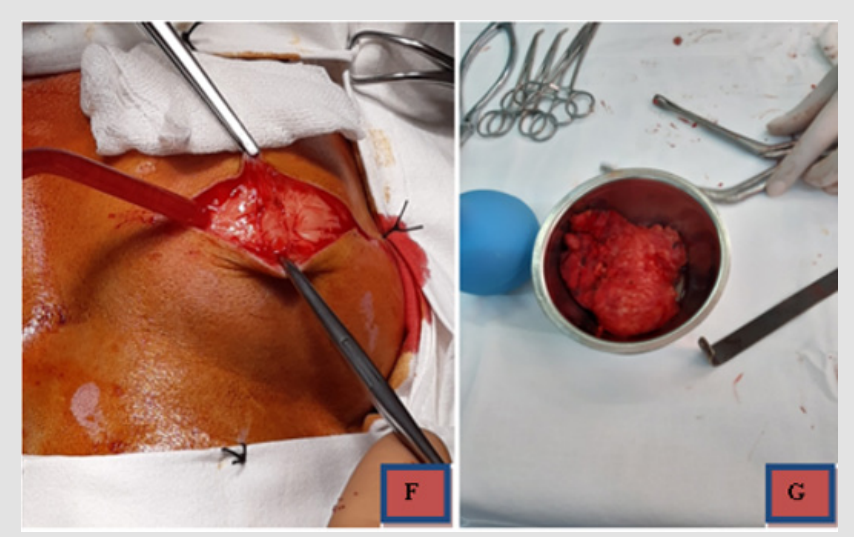

Figure 4:

F. Skin incision

G. Tumor macroscopic aspect.

No dissection plan has been found during the operation and the 2 masses were firmly attached to bone and periosteal membrane. The two masses have been sent to anatomo-pathological laboratory, which confirmed a great adypocytes proliferation. The masses were firmly attached to the occipito-nuchal region, hard in consistency and well encapsulated, slightly vascularized and with a macroscopical aspect compatible with adipos tissue overgrowth. The lesions were hard at touch and homogenous, it could be gripped with ease and it was detached from the bone mostly by hand. No significant hemorrage was produced. Hemostasis was performed with electro-coagulation and local hemostatic agents were used (Surgicel - oxycellulose). The two lesions had a maximal diameter of $10 \mathrm{~cm}$ (right) and $8 \mathrm{~cm}$ (left) (Figure 5). The two operations in a single step lasted about 3 hours. We did not use any drainage tube after the operation. The postoperative recovery was fast, with no signs of complications or need of re-intervention. At the end of the operation, the patient was admitted for a day in our Intensive Care Unit (ICU), for postoperative monitoring. The day after the surgery the patient was discharged from ICU, without any sign of complications. The patient was discharged from our hospital 7 days later, after the removal of the sutures. The skin became flat, with no sign of tumoral rests or damages to neighbour structures. At discharge, the patient was satisfied with the esthetical results 
and the two incisions were fully healed, with no signs of infection or tumoral rests. The whole hospitalization lasted 8 days. He was discharged with non-specific home treatment, predominently with non-steroidal anti-inflamatory drugs.

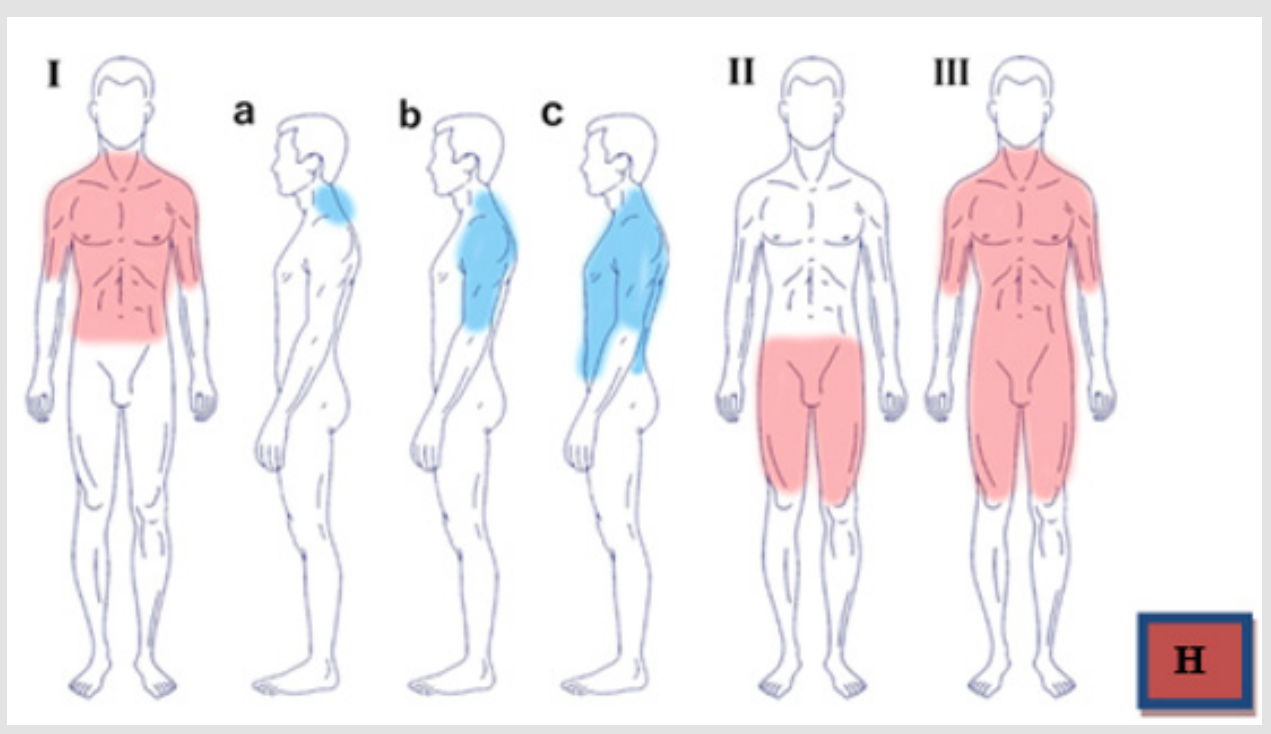

Figure 5: Madelung Disease classification.

\section{Discussion}

The MSL, or Madelung Disease, or Launois-Bensaude disease was described by Benjamin Brodie, in 1846 and was followed by the reports of Madelung (1898) and Launois and Bensaude (1899) and is now classified as a rare disease of fat metabolism. It includes a subcutaneous non-encapsulated fat tissue overgrowth which is painless and symmetrical. It mainly involves the head, neck, nuchal region, upper torso and eventually the limbs as well [1]. It is more prevalent in between the $3^{\text {rd }}$ and $5^{\text {th }}$ decade of life and is very prevalent in males (ratio $\mathrm{M}: \mathrm{F}=30: 1$ ) and its more common in Mediterranean area, showing a peak in the Italian population [2], as its incidence is it of 1:25000 in the general population. In 1984 Enzi proposed a classification of Madelung disease according to the clinical aspect and presentation of the lesions. A schematic summary is drawn above (Figure 6) [3].

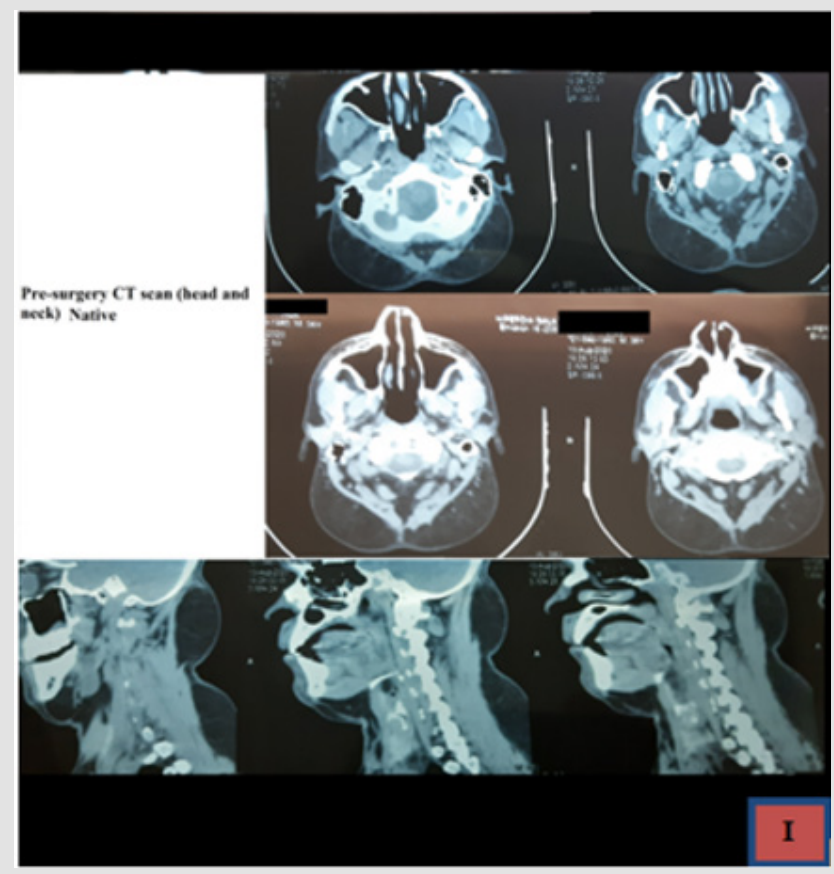

Figure 6: Pre-operative MRI scan of the nuchal region of the patient. 
Our case is classified as a type Ia Madelung disease, as it is shown in the pre-operative CT scans (Figure 1), with a bilateral mass over the posterior aspect of the nuchal region and the occipital bone. The other most common locations are visible in the table above. "The pathogenesis of this disease has not been completely defined, as such the abnormal proliferation of fat tissue is not related to an increase in caloric intake, but it looks like an active proliferation of the adipose tissue. The suggested-most mechanisms are defects in the respiratory chain or mutations/deletions in mitochondrial DNA" [4,5]. "Lipomatous fat deposits may originate from functionally defective brown adipous tissue and the tumoral fatty cells have an abnormal metabolic behaviour, which is characterized by a defect of the catecholamines acute lipolytic action. Some sort of alcoholic drinking disorder is present in more than $90 \%$, however our case did not show any sign of chronic alcoholic intake. A decrease in the beta-adrenergic receptors and subsequent defects in mitochondrial DNA may be the explication of pathogenic alcohol action over the fat tissue" [6]. "Even though that the history, epidemiology and clinical features are the basis of MLS diagnosis [7] in some cases may be challenging, due to the common arise of obesity in the general population $(1 ; 4)$. Moreover, such patients usually presents with other significant commorbidities, such as type II diabetes mellitus, glucose intolerance or hyperuricemia. Diseases associated with chronic alcoholic consumption (macrocytic anemia, hepatopathy, periferal neuropaties) are very common is MSL" [7]. Our patient did not show any sign of liver suffering or chronic alcoholic consuption associated diseases. Although alcohol withdrawal and weight loss are recommended, these measures are not effective to reverse/ stop the progression of the tumors. Currently, surgical treatment is the only option available, and so the fat masses removal remains the only therapy of choice. However the overall recurrence rate is describe as up as $63 \%$ of cases [7].

\section{Conclusion}

Despite the high recurrency rate (63\%) [6] the surgical approach is the only current treatment of choice for these benign lesions. The surgery is quite challenging if complications arise (like odynophagia, dysphagia or cervical vein inclusions) but usually has a simple route, as the surgery is performed extracranially. Neurosurgeons may approach such lesions due to the their vecinity with other compartments of the neuro-axis. However, high volume lesions may be quite difficult to be approached and fully removed. In the medical literature, cases with lesions up to kilograms in weight have been described, with succesful surgical removal. Hence, is very important to give a surgical treatment to such cases, even though the lesions are not painful. Social stigma and ostracizing of such patient have been described, including difficulties at acquiring and/or mantaining a job, which make the surgical option practically mandatory. The high rate of recurrency should not stop us from giving them the only current therapy of choice. A good esthetical and functional result (Figure 7) after surgery is possible, as shown in our patient. The patient signed all the informed consent declarations to allow this publication of his case.

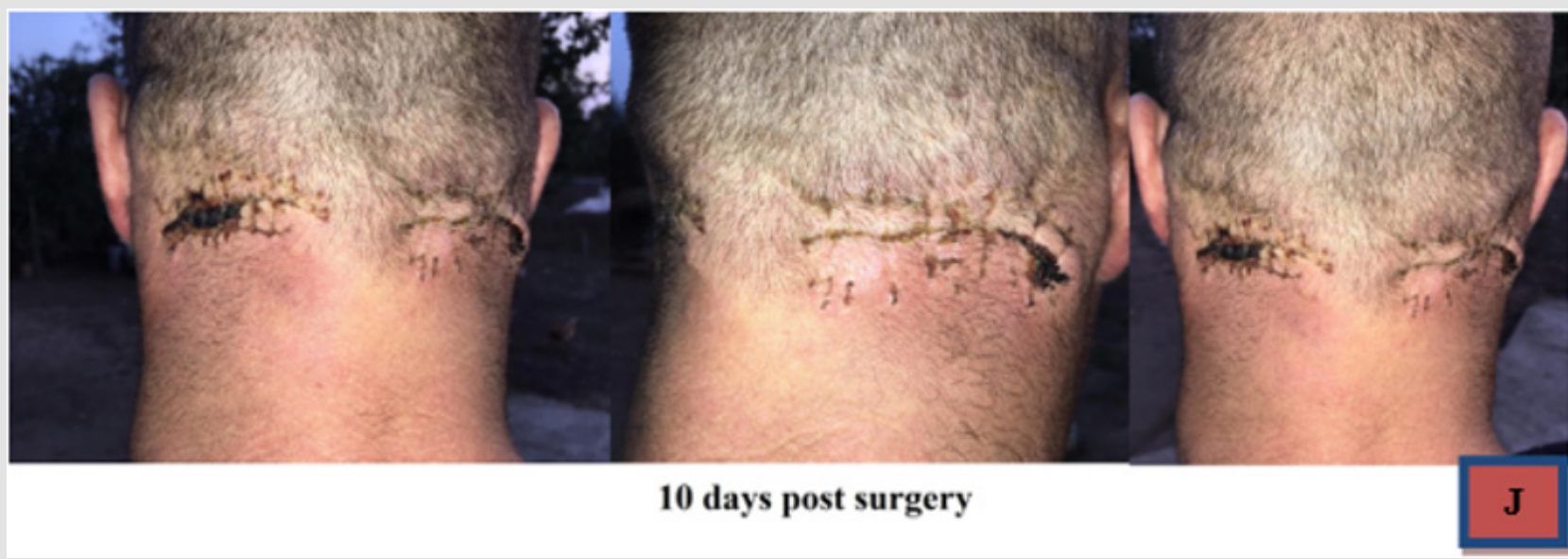

Figure 7: Post-surgical skin aspect.

\section{Final Discussion}

The Madelung disease is a rare clinical entity [8]. Despite the high chance of recurrence, its malignant potential is quite low. Its implications in the patient's life are important and surgery remains the only treatment of choice. Weight loss and alcohol withdrawl are indicated, however they dont stop the adypocytes proliferation. There is a suggested pathway of deletions and mutations over the mitochondrial DNA. Hence, it is not an energy intake related proliferation, but rather an uncontrolled metabolic behaviour. It is challenging to diagnose such a disease, with the rapidly increasing obesity frequency. As differential diagnostics, Cushing disease, Frölich syndrome and lymphoma must be considered. Usually these patients show signs of chronic alcoholic consumption (eg hepatopathy or macrocytic anemia) or metabolic disorders (DM type II, obesity) in up to $90 \%$ of cases [9]. 


\section{References}

1. Tadisina KK, Mlynek KS, Hwang LK, Riazi H, Papay FA, et al. (2015) Syndromic lipomatosis of the head and neck: a review of the literature. Aesthetic Plast Surg 39(3): 440-448.

2. Enzi G, Busetto L, Sergi G, Coin A, Inelmen EM, et al. (2015) Multiple symmetric lipomatosis: a rare disease and its possible links to brown adipose tissue. Nutr Metab Cardiovasc Dis 25(4): 347-353.

3. Schiltz D, Anker A, Ortner C, Tschernitz S, Koller M, et al. (2018) Multiple symmetric lipomatosis: new classification system based on the largest German patient cohort. Plast Reconstr Surg Glob Open 6(4): e1722.

4. Plummer C, Spring PJ, Marotta R, Chin J, Taylor G, et al. (2013) Multiple Symmetrical Lipomatosis--a mitochondrial disorder of brown fat. Mitochondrion 13(4): 269-276.

ISSN: 2574-1241

DOI: 10.26717/BJSTR.2021.33.005397

Boghi David M. Biomed J Sci \& Tech Res

(c) (P) This work is licensed under Creative

Submission Link: https://biomedres.us/submit-manuscript.php
5. Suresh Chandran CJ, Godge YR, Oak PJ, Ravat SH (2009) Madelung's disease with myopathy. Ann Indian Acad Neurol 12(2): 131-132.

6. Pinto CI, Carvalho PJ, Correia MM (2017) Madelung's Disease: Revision of 59 Surgical Cases. Aesthetic Plast Surg 41(2): 359-368.

7. Zuber M, Pittasch D (2006) Benign symmetric lipomatosis (LaunoisBensaude syndrome). A rare cause of muscular weakness. Eur J Med Res 11(4): 174-177.

8. Maximiano LF, Gaspar MT, Nakahira ES (2018) Madelung disease (multiple symmetric lipomatosis). Autops Case Rep 8(3): e2018030.

9. Shibasaki ÍI, Shibasaki HI, Nakamoto Tde S, Baccan FS, Raposo LS (2014) Multiple symmetrical lipomatosis (Madelung's disease). Braz J Otorhinolaryngol 80(1): 90-91.

$\begin{array}{ll}\text { BIOMEDICAL } & \text { Assets of Publishing with us } \\ \text { RESEARCHES } & \text { - Global archiving of articles } \\ \text { - Immediate, unrestricted online access } & \text { - Rigorous Peer Review Process } \\ & \text { - Authors Retain Copyrights } \\ \end{array}$

